\title{
Persistent anti-tumor effects via recombinant adeno-associated virus encoding herpes thymidine kinase gene monitored by PET-imaging
}

\author{
JI YUN KIM ${ }^{1}$, JI HYUN KIM ${ }^{1}$, MARIA KHIM ${ }^{4}$, HAN SAEM LEE ${ }^{1}$, JIN HWA JUNG ${ }^{3}$, \\ DAE HYUK MOON ${ }^{2}$, SUNJOO JEONG ${ }^{5}$ and HEUIRAN LEE ${ }^{1,4}$ \\ Departments of ${ }^{1}$ Microbiology, ${ }^{2}$ Nuclear Medicine, ${ }^{3}$ Molecular Imaging, ${ }^{4}$ Biomedical Institute \\ of Technology, University of Ulsan College of Medicine; ${ }^{5}$ Department of Molecular Biology, \\ College of Natural Sciences, Dankook University, Seoul, Republic of Korea
}

Received October 5, 2010; Accepted January 27, 2011

DOI: 10.3892/or.2011.1190

\begin{abstract}
Despite the well-documented advantages of the recombinant adeno-associated virus (rAAV) as a gene delivery vehicle, including its non-pathogenic and long-term therapeutic gene expression, there have been very limited studies on its potential for producing persistent anti-tumor effects, particularly in vivo. To address this issue, we constructed $\mathrm{rAAV}$ vectors encoding herpes simplex virus 1-thymidine kinase (HSV-TK) or its mutant form (sc39TK) as therapeutic genes, and GFP as a control gene. Effective rAAV-mediated gene delivery was readily observed in human cancer cells using immunocytochemistry and Western blotting. Cell survival analysis following prodrug ganciclovir treatment implied that both preferential and superior cytotoxicity was achieved by rAAV-sc39TK introduction. Persistent anti-tumor effects in vivo were investigated in Balb/c nude mice bearing human cancer cells treated with either rAAV-sc39TK or -GFP. Severe tumor growth inhibition was clearly observed only in the case of sc39TK with ganciclovir treatment. Non-invasive micro-PET imaging using ${ }^{18} \mathrm{~F}-\mathrm{FHBG}$ directly correlated with persistent anti-tumor effects by sc39TK. Therefore, the present study provides evidence that rAAV-mediated persistent therapeutic gene expression can occur, resulting in long-term anti-tumor activities and that these events can be readily monitored using micro-PET imaging.
\end{abstract}

\section{Introduction}

Viral vectors are advantageous due to their high transduction efficiencies and have consequently been utilized as efficient gene delivery vehicles in cancer gene therapy. Among them, recom-

Correspondence to: Dr Heuiran Lee, Department of Microbiology, University of Ulsan College of Medicine, 86 Asanbyeongwon-gil, Songpa-gu, Seoul 138-736, Republic of Korea

E-mail: heuiran@amc.seoul.kr

Key words: recombinant adeno-associated virus, HSV-TK, ganciclovir, anti-tumor efficacy, long-term gene expression, micro-PET binant adenovirus (rAd) has been the most commonly used viral vector (1). However, the utility of rAd is limited by its shortterm gene expression, which may not be ideal for treating metastatic or recurrent malignant tumors. As opposed to rAd, recombinant adeno-associated virus (rAAV) is non-pathogenic and can also achieve long-term expression of transgenes, which lends advantages in a long-term therapeutic model (2). Thus, rAAV vectors have been employed in many clinical trials aiming for persistent therapeutic effects in cases such as cystic fibrosis, hemophilia B, and neurological diseases (3). In recent studies, rAAV vectors have even emerged as an alternative platform for cancer gene therapy (4). Among the various rAAV serotypes, rAAV2 serotype has been commonly used for cancer gene therapy because of its strong tropism towards tumor cells $(5,6)$. rAAV2 mediates high transgene expression selectively in tumor cells by use of its tumor-associated receptors such as heparin sulfate, EGFR (7), and HGFR (8). Yet, there have been very limited studies on the potential of rAAV vectors to achieve persistent anti-tumor effects, particularly in vivo.

Various therapeutic genes are employed in cancer gene therapy, including tumor suppressors, anti-angiogenesis agents, and apoptosis inducers (9). Among these, herpes simplex virus 1-thymidine kinase (HSV-TK) is used as a suicide agent in combination with prodrug ganciclovir (GCV) to achieve cytotoxic therapy (10). First, HSV-TK expression is induced in tumor cells. Then tumor cells are treated with GCV, which is phosphorylated by HSV-TK. The phosphorylated GCV inhibits DNA replication and selectively induces cell death in HSV-TK expressing tumor cells. Another advantage of HSV-TK/GCV therapy is the 'bystander effect', which increases the efficiency of killing untransduced neighboring tumor cells (11). Recently, alternative mutant genes have been generated through semi-random mutagenesis of HSV-TK to preferentially improve affinity to GCV and circumvent GCV cytotoxicity (12). Mutant sr39TK is created by a five codon substitution from HSV-TK (13). Then, silent mutations are introduced to GCV-resistant spliced acceptor and donor sequences to derive the mutant sc39TK. As a splice-corrected version of sr39TK, mutant sc39TK abrogates aberrant splicing of the HSV-TK gene and increases its enzymatic activity (14). 
HSV-TK and its mutant alternatives also hold promise as a molecular imaging tool for tracking the metastasis and growth of cancer cells (15-17). Besides drug-substrate GCV, HSV-TK and its mutant alternatives can specifically phosphorylate other molecules including various radioactive probes such as ${ }^{18} \mathrm{~F}-\mathrm{FHBG}$ and ${ }^{124} \mathrm{I}-\mathrm{FIAU}$ (18-20). These phosphorylated probes accumulate in cancer cells expressing HSV-TK and provide a method to visualize tumor growth through micro-PET. This non-invasive visualization method can be used to closely monitor tumor growth by multiple imaging sessions throughout a prolonged time period. HSV-TK-mediated phosphorylation of these radioactive probes provides a way to measure, characterize, and quantify anti-tumor effects. However, this imaging technology has not yet been combined with the advantages of rAAV vector systems.

Thus, we attempted to examine persistent anti-tumor effects in Balb/c nude mice bearing human tumor cells treated with rAAV2-HSV-TK and simultaneously monitored its efficacy using micro-PET scanning. As a hyperactive variant of the suicide agent HSV-TK, transgene sc39TK was also hired and combined with the drug substrate GCV to achieve cytotoxic genetic therapy selectively in tumor cells. The rAAV2 vector system explored in this study will thus prove the utility of rAAV vectors in inducing persistent anti-tumor effects and providing a valuable molecular monitoring tool for cancer development.

\section{Materials and methods}

Cell culture. Human cancer cell line, HeLa cells, was purchased from the American Type Culture Collection (ATCC; Manassas, VA). These cell lines were maintained in Dulbecco's modified Eagle's medium supplemented with $10 \%$ fetal bovine serum, L-glutamine $(2 \mathrm{mM})$, penicillin $(100 \mathrm{IU} / \mathrm{ml})$ and streptomycin $(50 \mu \mathrm{g} / \mathrm{ml})$ in a $5 \% \mathrm{CO}_{2}$ incubator at $37^{\circ} \mathrm{C}$.

Viral plasmid construction and recombinant adenoassociated virus (rAAV) preparation. $\mathrm{pSp} 72$ rAAV2-GFP was constructed by blunt-end ligation of the $2.3-\mathrm{kb} P v u \mathrm{II}-P v u \mathrm{II}$ fragment of pHpa-trs-SK into the BglII and HindIII sites of the pSp72 vector, thus it contains the full length recombinant AAV2 genome with the GFP protein sequence encoded. pHpatrs-SK was received as a gift from Dr M. McCarty (Ohio State University, USA). pSp72-scAAV2-sc39TK was constructed by subcloning the BamH-SalI PCR fragment of sc39TK gene from pcDNA3.1(+)-sc39TK into BamH-SalI sites in pSp72scAAV2-GFP from which the corresponding fragment was removed. Therefore, the final product was the mutant sc39TK cassette in rAAV2 genome. The generation, preparation, and titration of the rAAV2 vector were performed according to the method outlined previously (21).

Immunocytochemistry and Western blot analysis. HeLa cells were seeded in 48-well and 6-well plates for immunocytochemistry and Western blot analysis, respectively. Twenty-four hours after seeding, the cells were infected with rAAV2sc39TK at various MOIs. Subsequent experimental procedures were conducted as outlined in previous studies, generally on day 2 post-infection $(22,23)$. For this process, the primary antibodies HSV-TK (4C8) and actin were purchased from
William Summers (Yale University, USA) and Sigma (St. Louis, $\mathrm{MO})$, respectively.

Cytotoxicity assay. HeLa cells seeded in a 6-well plate were infected with rAAV2 vectors encoding GFP, wild-type TK, or mutant sc39TK. Twenty-four hour post-infection, cells were seeded in a 96-well plate. The GCV (Cymevene, Roche, Germany) was applied in various concentrations of $0,0.001$, $0.01,0.05,0.1,0.5$, and $1 \mu \mathrm{g} / \mathrm{ml}$ in DMEM complete media and cells were incubated in these various GCV treatment solutions for another 5 days. Surviving cells were stained with crystal violet solution in 50\% methanol for $1 \mathrm{~h}$ and then washed extensively with distilled water before having their absorbance read at $540 \mathrm{~nm}$ with a microplate spectrophotometer (Spectra MAX 340pc, Molecular Devices, Sunnyvale, CA, USA). The percentage cell survival was calculated by the following equation: \% cell survival $=$ (the absorbance in experimental group - the absorbance in blank) / (the absorbance in control group - the absorbance in blank) x100.

Generation of tumor models and GCV treatment. Each Balb/c nude mice (male, 7 weeks) was subcutaneously inoculated in the right and left forelimbs with $5 \times 10^{6}$ of rAAV2-sc39TKinfected HeLa cells and sham-treated HeLa cells, respectively. With the approval of the University committee on the use and care of animals, mice were maintained in an optimal temperature and humidity-controlled room with a $12 \mathrm{~h}$ light/dark cycle. Sham-treatment included mock-treated tumors or GFPexpressing tumors. Tumor size was measured using a caliper, and tumor volume was calculated according to the formula $(\pi / 6) \times(a) \times(b) \times(c)$; (a) tumor dimensions including the largest diameter, (b) the perpendicular diameter, and (c) the vertical diameter. When the tumors had reached a volume of $\sim 40 \mathrm{~mm}^{3}$ ( 9 days post inoculation), half of the mice were intraperitoneally injected with GCV $10 \mathrm{mg} / \mathrm{kg} /$ day for 16 days. The other half was intraperitoneally injected with equivalent amounts of saline. Tumor size and volume were measured every 2 to 3 days up until day 45 post infection.

Micro-PET image acquisition. When the tumors had reached a volume of $\sim 100 \mathrm{~mm}^{3}$ (14-15 days post inoculation), the small animal scanner micro-PET was used to acquire tomographic whole-body images of the biodistribution of ${ }^{18} \mathrm{~F}$-FHBG (R4, Concord Microsystems, Knoxville, TN, USA). After mice were anesthetized, they were injected with approximately $200 \mu \mathrm{Ci}(\sim 3.7 \mathrm{MBq})$ of ${ }^{18} \mathrm{~F}-\mathrm{FHBG}$ via the tail vein about $1 \mathrm{~h}$ prior to the start of micro-PET data acquisition. Prior to positioning the mice for PET scans, their bladders were emptied as much as possible. Imaging data for each mouse was acquired during $10 \mathrm{~min}$. For image reconstruction, list-mode data was sorted into three-dimensional sonograms. Region of interest (ROI) analyses of reconstructed images were done by vendor software (ASI Pro 1.1, Concord Microsystems) (24).

\section{Results}

In vitro cytotoxicity by rAAV2-mediated $T K$ expression and GCV treatment. Various AAV2 vectors were constructed bearing wild-type HSV-TK (rAAV2-TK), mutant sc39TK (rAAV2-sc39TK), and GFP (rAAV2-GFP) as control (Fig. 1A), 
A

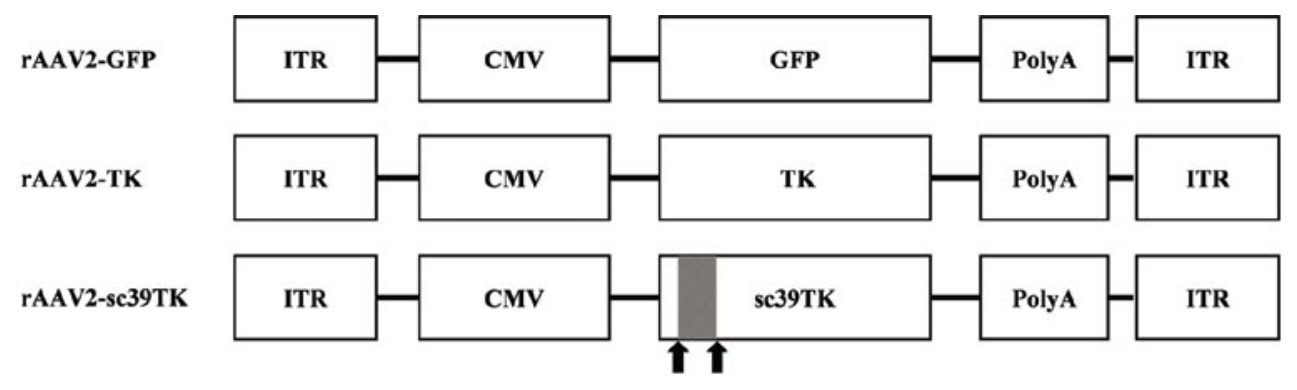

B
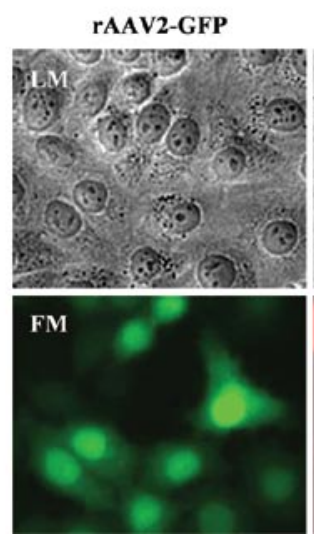

rAAV2-TK
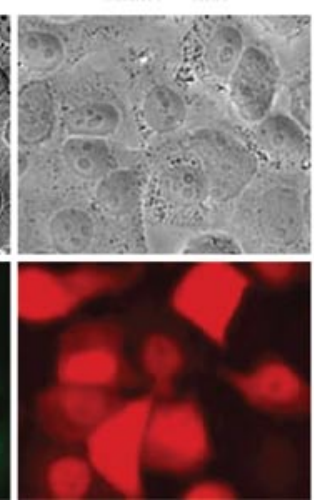

rAAV2-sc39TK

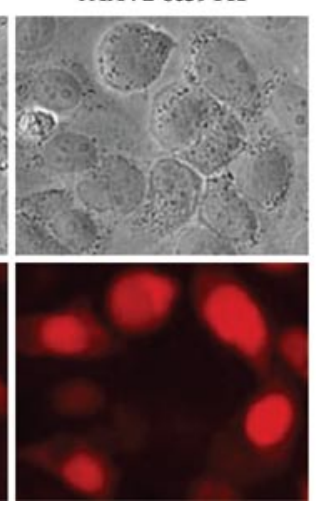

C

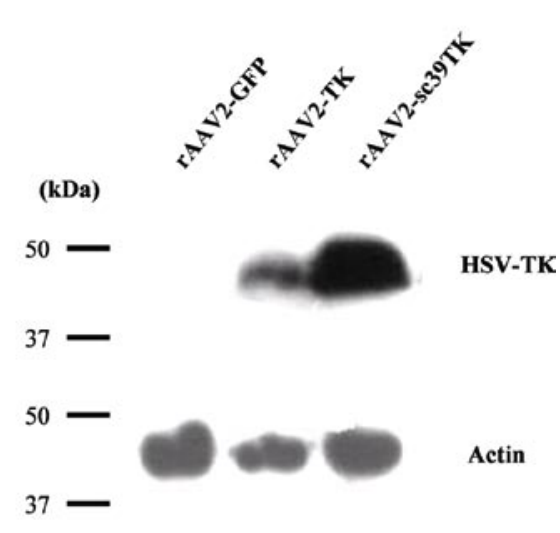

Figure 1. Features of rAAVs in the present study and TK-based transgene expression in HeLa cells. HeLa cells were infected with rAAV2-wild-type TK, rAAV2-sc39TK, or rAAV2-GFP at MOI=1,000. At $48 \mathrm{~h}$ post-infection, the cells were recovered to evaluate transduction efficiencies. The expression of transgenes was confirmed by immunocytochemistry and Western blotting. (A) Schematic representations of rAAV2 vectors. The sc39TK is derived by introducing two silent point mutations in the cryptic splicing donor and acceptor sites of the gene (indicated by arrow heads) (14). (B) GFP expression images under FM and immunocytochemistry images specific to TK. (C) Western blot analysis for TK. The data shows efficient expression of rAAV2-delivered GFP, TK, and sc39TK in the cells. ITR, inverted-terminal repeat; CMV, CMV promoter; LM, light microscopy; FM, fluorescent microscopy.

and transduction efficiencies were determined in HeLa cells following virus treatment. Immunocytochemistry and Western blotting of TK expression, along with GFP expression, suggested effective rAAV2-mediated delivery of all transgenes into the cells (Fig. 1B and C). At MOI 1000, almost entire cell populations were transduced.

In cytotoxicity assays, GFP-expressing cells experienced no significant cytotoxicity while sc39TK-expressing HeLa cells suffered from dramatic cytotoxicity that further increased with higher GCV doses (Fig. 2A and B). LD $_{50}$ in sc39TKexpressing HeLa cells was $0.01 \mathrm{GCV}$ concentration. $\mathrm{LD}_{50}$ in HSV-TK was $1.0 \mathrm{GCV}$ concentration, which was 100 times less sensitive than that by sc39TK (data not shown). Crystal violet staining taken at GCV concentration of $0.01 \mu \mathrm{g} / \mathrm{ml}$ clearly showed drastically greater cell death in sc39TK-expressing cells in comparison to GFP-expressing cells (Fig. 2C). Further experiments conducted in a different human cancer cell line, SK-Hep1, also demonstrated successful transgene delivery and cytotoxicity to cancer cells by both rAAV2-TK and sc39TK with GCV treatment. Again, selective cytotoxicity by sc39TK was much greater over HSV-TK in SK-Hep1 (data not shown).

In a long-term study to verify persistent transgene expression by rAAV2, significant levels of rAAV2-mediated transgene expression in HeLa cells was detected over a month post infection (data not shown). Thus, these in vitro results support the persistent therapeutic potential of rAAV2-sc39TK to achieve long-term transgene expression and cytotoxicity in various human cancer cell lines.

Persistent tumor growth inhibition by rAAV2-sc39TK/GCV treatment in vivo. To examine anti-tumor effects by rAAV in vivo, nude mice were implanted with rAAV2-sc39TK or sham-treated HeLa cells as demonstrated in Fig. 1, and intraperitoneally administered with GCV $(n=4)$ or saline $(n=4)$. As expected, severe tumor growth retardation was observed in all sc39TK-expressing tumors in the presence of GCV (Fig. 3A). In one tumor out of these four cases, complete regression was observed. No significant growth inhibition was observed in sc39TK-expressing tumors without GCV treatment. In contrast, sham-treated tumors showed no significant growth inhibition not only without GCV but with GCV treatment as well. Accordingly, $H \& E$ staining indicated that massive necrotic region resided only in the sc39TK-GCV group (Fig. 3B). Taken all together, these data demonstrated that sc39TK/GCV cyto-toxic therapy via rAAV2 could achieve persistent anti-tumor effects in vivo.

Micro-PET imaging of tumors expressing TK via rAAV2. To non-invasively monitor anti-tumor effects by rAAV, ${ }^{18} \mathrm{~F}-\mathrm{FHBG}$, a radioactive probe that interacts with sc39TK (data not shown), was systematically injected in the mice as described in Fig. 3. Micro-PET imaging was then conducted from $1 \mathrm{~h}$ up to 4 weeks post tumor implantation. In micro-PET images at 
A
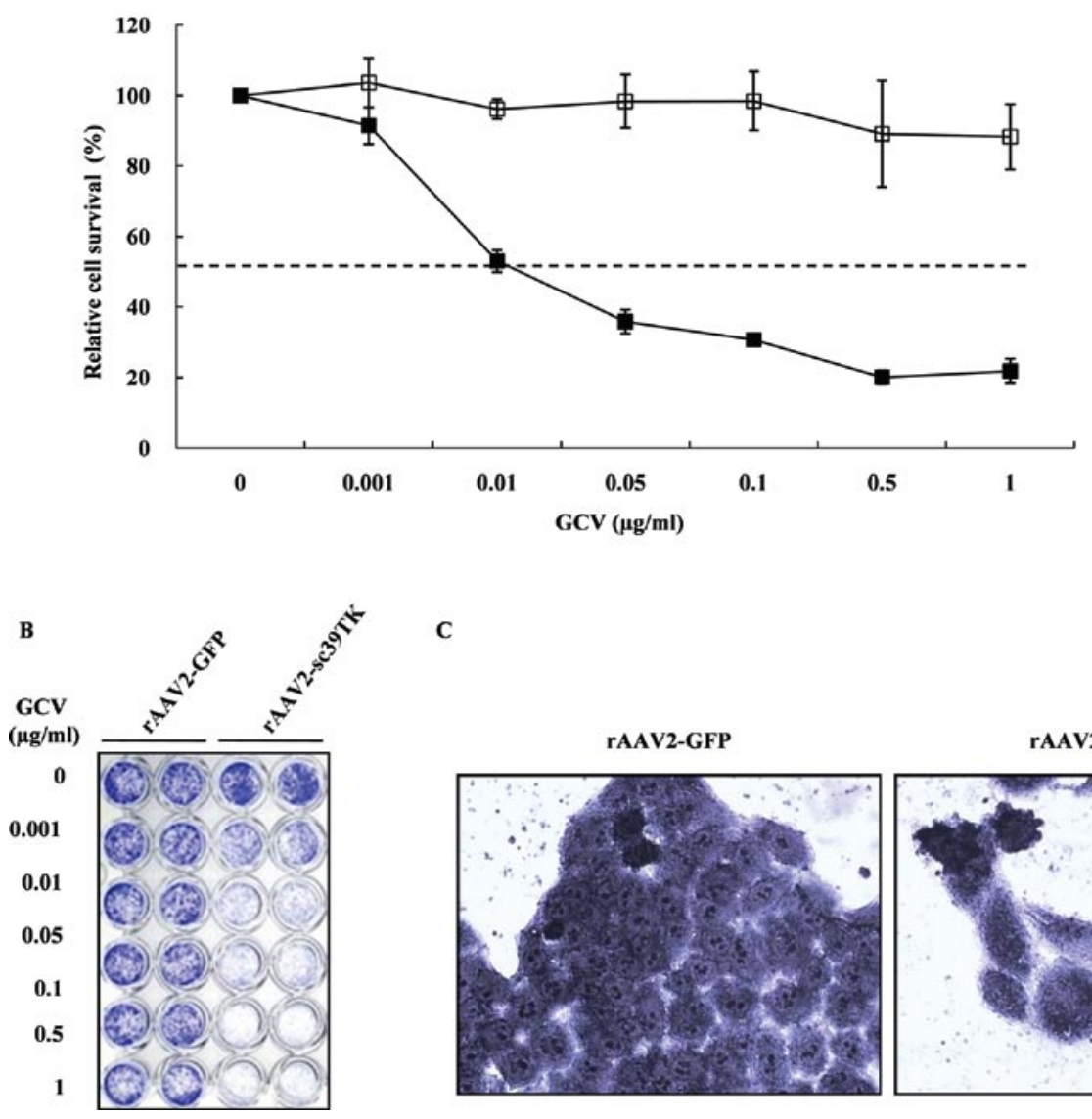

C
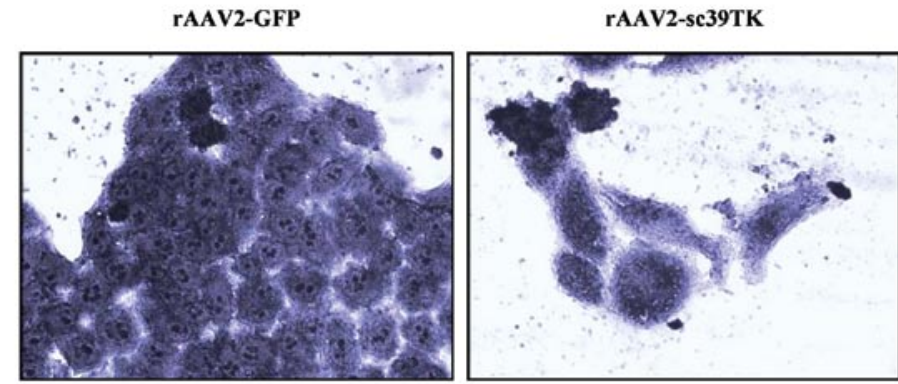

Figure 2. Enhanced cytotoxicity of rAAV2-sc39TK in HeLa cells. (A) Cells were infected with rAAV2-sc39TK at MOI 1,000 (匚). As control, cells were infected with rAAV2-GFP at the same MOI ( $\square$ ). sc39TK with GCV treatment showed significant cytotoxicity effects, whereas control GFP showed no significant cytotoxicity effects regardless of GCV treatment. (B) Crystal violet-stained image of surviving cells in a 96-well. (C) Crystal violet-stained cells at a higher magnification under GCV $0.01 \mu \mathrm{g} / \mathrm{ml}$. The data depict the increasing cytotoxicity efficacies of sc39TK with increasing GCV concentrations.

2 weeks, prior to GCV administration, the sc39TK-expressing tumors showed significant signaling of ${ }^{18} \mathrm{~F}-\mathrm{FHBG}$ compared to the sham-treated tumors (Fig. 4). Subsequently, with GCV treatment, tumors expressing sc39TK showed significantly decreased signaling of ${ }^{18} \mathrm{~F}$-FHBG. These observations of specific reduction in ${ }^{18} \mathrm{~F}-\mathrm{FHBG}$ signaling, along with $\mathrm{HeLa}$ tumor growth retardation in the sc39TK-GCV group, demonstrated that tumor growth inhibition following GCV treatment can be monitored via non-invasive imaging. Contrary to the absence of signaling in sham-treated tumors at the initial stages of tumor development, some degree of non-specific signaling were observed in the subsequent weeks as the tumor sizes became greater. These data thus indicated that the interactions of sc39TK and ${ }^{18} \mathrm{~F}-\mathrm{FHBG}$, captured by micro-PET, can serve as an effective imaging tool to monitor the anti-tumor effects of sc39TK/GCV treatment, with some limitation.

\section{Discussion}

Here, we explored i) the potential of rAAV as a gene delivery tool driving persistent anti-tumor effects by hiring mutant HSV-TK and ii) the feasibility of TK $/{ }^{18} \mathrm{~F}-\mathrm{FHBG}$-based microPET imaging for non-invasively monitoring the anti-tumor effects. By delivering rAAV2-sc39TK and GCV together to human cancer cells, we demonstrated that long-term anti-tumor effects can be obtained both in vitro and in vivo. These preferential anti-tumor effects were readily captured repeatedly using noninvasive micro-PET.

When sham- and rAAV2-sc39TK-treated HeLa tumors were implanted in Balb/c nude mice, dramatic GCV-induced cell death was observed in sc39TK-expressing tumors with one case of total tumor regression. Anti-tumor effects of rAAV-mediated sc39TK expression and GCV were monitored and confirmed for several weeks post tumor implantation and affirmed the expected advantage of employing persistent transgene expression by rAAV. Other studies have also employed rAAV to establish various long-term therapeutic strategies. For example, in one study, rAAV-mediated dopamine expression was induced in MPTP-lesioned primate brains as a potential therapeutic method for Parkinson patients. Consequently, rAAV-mediated transgene expression was sustained for 6 years in vivo and successfully achieved long-term improvement in clinical rating scores (25). In a more recent study, rAAVmediated microRNA delivery to hepatocellular carcinoma cells in an in vivo model successfully produced stable transgene expression and tumor-suppressant activity (26). The results of these previous studies in addition to our own support the advantages of rAAV mediated long-term therapeutic effects and the potential of rAAV as a promising and important tool for gene therapy. 

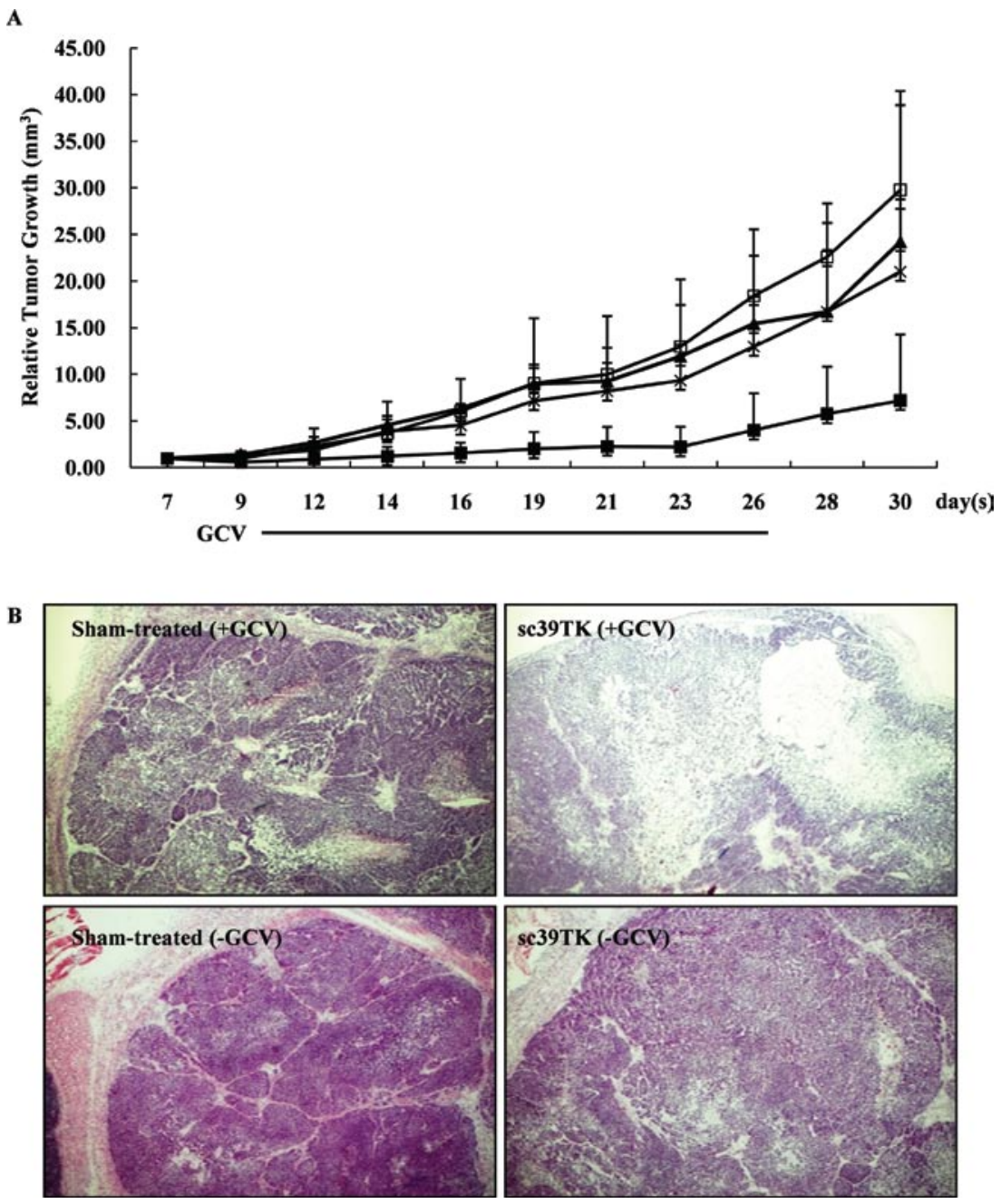

Figure 3. HeLa tumor growth suppression by sc39TK expression and GCV treatment in mice. (A) After being implanted with rAAV2-treated cells, the Balb/c nude mice were intraperitonally treated with GCV. The size of tumors expressing sc39TK was significantly reduced following GCV treatment. By contrast, tumors with sham-treatment showed no significant inhibition of growth. (B) H\&E staining of tumors harvested from mice at 5 weeks post tumor implantation.

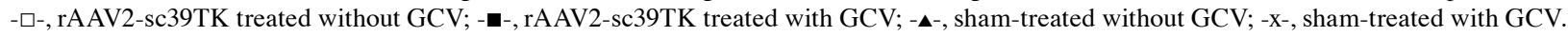

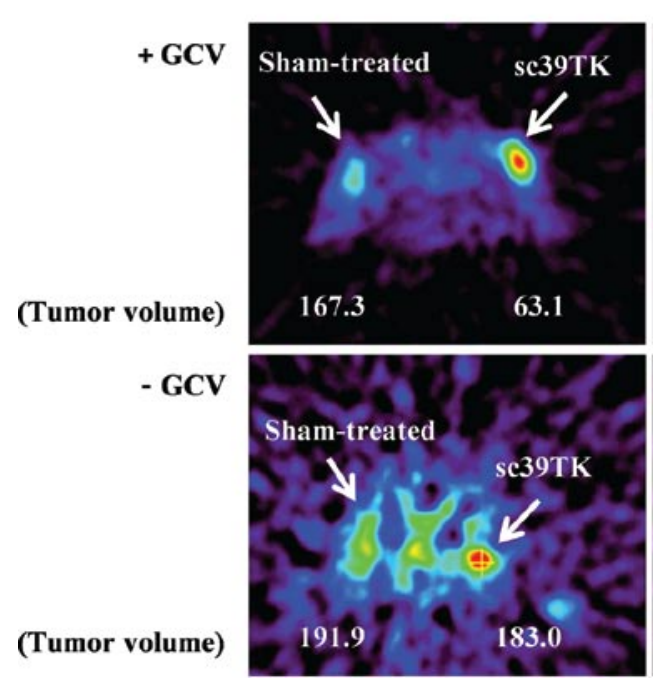

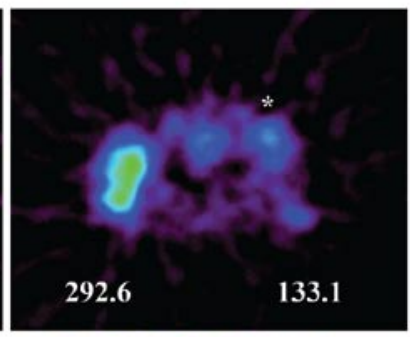

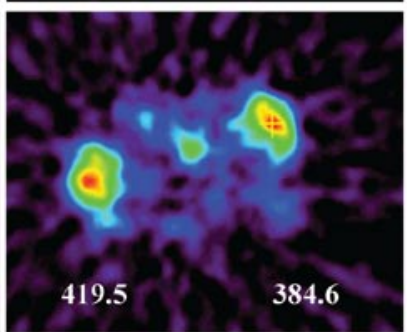

4
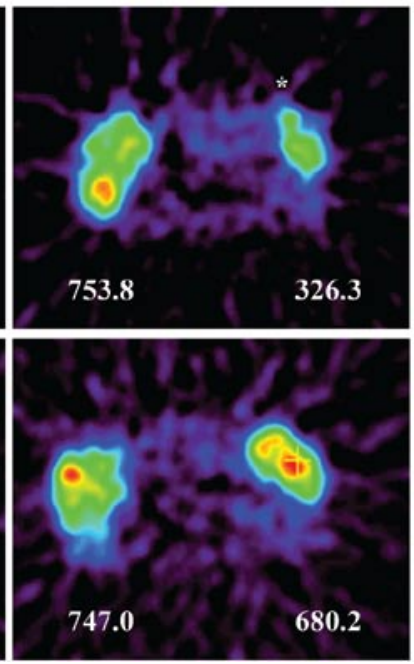

High

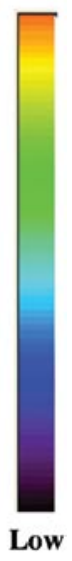

Figure 4. Non-invasive micro-PET analysis of rAAV2-treated tumors. PET-scans were performed on nude mice after intravenous injection of ${ }^{18} \mathrm{~F}$-FHBG. Multiple images were obtained from the same mice representing each experimental group. Imaging data indicate that the signal of ${ }^{18} \mathrm{~F}$-FHBG correlated well with the degree of tumor growth retardation in sc39TK-GCV tumors (*). 
In vitro functional studies provided clear evidence that effective transgene expression and significant cytotoxicity can be achieved by rAAV-mediated sc39TK expression and GCV treatment in human cancer cells. sc39TK, the mutant form of HSV-TK was reported as a more optimal alternative because it could further induce greater anti-tumor cytotoxicity with GCV by retaining higher enzymatic activity due to not generating aberrant splicing forms (14). To confirm whether sr39TK is indeed invulnerable to spontaneously generating alternative splicing forms, we infected various human cancer cell lines with rAAV2-sr39TK or -TK, including FADU, U251, U81-MG, MDA-MB345, TE3, A541, Baxpc3, and SK-Hep1. RNAs extracted from these cells were amplified by RT-PCR using TK-specific primers that allowed the generation of various splicing forms. Both agarose gel analysis and DNA sequencing analysis suggested that only enzymatically functional splicing forms were produced by both genes (data not shown) (27). However, an in vivo situation might lend different results and our own study nevertheless demonstrated the potential of mutant sc39TK for effective GCV-induced gene therapy.

Presently, we also explored the application of rAAV for the first time in micro-PET imaging as a non-invasive, efficient method to monitor tumor development. Micro-PET imaging using ${ }^{18} \mathrm{~F}-\mathrm{FHBG}$ also provided further visual evidence of the tumoral growth inhibition by sc39TK/GCV treatment (28). Radioactive probe ${ }^{18} \mathrm{~F}-\mathrm{FHBG}$ was delivered and its interactions with rAAV-mediated sc39TK expression were captured via micro-PET imaging, which visualized the anti-tumor effects by sc39TK and GCV over multiple imaging sessions. In addition to the probe used in this study, there are a wide variety of probes that have been developed, such as ${ }^{18} \mathrm{~F}$-FEAU (29), and ${ }^{123}$ I-FIAU (30), that vary in their safety and their selectivity and affinity to host kinases. In our study, we employed ${ }^{18} \mathrm{~F}-\mathrm{FHBG}$ for its high selectivity for HSV-TK. However, as further investigations of radioactive probes continue, it would be beneficial to keep in mind the various options to optimize the specificity and efficacy of micro-PET imaging techniques.

Our study was limited by the small number of in vivo models which prevented significant quantitative analysis of micro-PET images. Thus, further investigation with additional tumor xenograft experiments using micro-PET and ${ }^{18} \mathrm{~F}-\mathrm{FHBG}$ tracer with larger in vivo experimental groups could provide additional quantitative evidence for rAAV-sc39TK/GCV therapy. Eventually, long-term in vivo models employing rAAV2sc39TK could be developed to address the possibility of therapeutic efficacy for metastatic tumors. The anti-metastasis efficacy achieved by rAAV vectors encoding other antiangiogenic transgenes, such as TIMP1 (31), endostatin (32-34), VEGFR antibody, and vasostatin has already been shown in past experiments (35). Thus, future studies investigating rAAV2-sc39TK in long-term in vivo models would be the next important step to support both the anti-tumor and anti-metastatic effects of sc39TK/GCV therapy.

In conclusion, our study demonstrated that $\mathrm{rAAV}$ vectors bearing sr39TK can be employed as efficient tools for antitumor efficacy in long-term cancer gene therapy and for monitoring tumor development using micro-PET imaging. As afore-mentioned, future studies of rAAV-sc39TK in long-term in vivo metastatic models with rigorous statistical analyses of micro-PET imaging would provide even greater support for
rAAV and sc39TK/GCV as promising therapeutic tools in cancer gene therapy.

\section{Acknowledgements}

This study was partly supported by grants from the Korea Science and Engineering Foundation (M1053404002; S. Jeong) and the Ministry of Health and Welfare (A062254; H. Lee), Republic of Korea.

\section{References}

1. Shirakawa T: The current status of adenovirus-based cancer gene therapy. Mol Cells 25: 462-466, 2008.

2. Li C, Bowles DE, van Dyke T and Samulski RJ: Adenoassociated virus vectors: potential applications for cancer gene therapy. Cancer Gene Ther 12: 913-925, 2005.

3. Mueller $\mathrm{C}$ and Flotte TR: Clinical gene therapy using recombinant adeno-associated virus vectors. Gene Ther 15: 858-863, 2008.

4. Davidoff AM, Nathwani AC, Spurbeck WW, Ng CY, Zhou J and Vanin EF: rAAV-mediated long-term liver-generated expression of an angiogenesis inhibitor can restrict renal tumor growth in mice. Cancer Res 62: 3077-3083, 2002.

5. Buning H, Ried MU, Perabo L, et al: Receptor targeting of adeno-associated virus vectors. Gene Ther 10: 1142-1151, 2003.

6. Burger C, Nash K and Mandel RJ: Recombinant adeno-associated viral vectors in the nervous system. Hum Gene Ther 16: 781-791, 2005.

7. Ho DT, Wykoff-Clary S, Gross CS, et al: Growth inhibition of an established A431 xenograft tumor by a full-length anti-EGFR antibody following gene delivery by AAV. Cancer Gene Ther 16: 184-194, 2009.

8. Smith AD, Collaco RF and Trempe JP: Enhancement of recombinant adeno-associated virus type 2-mediated transgene expression in a lung epithelial cell line by inhibition of the epidermal growth factor receptor. J Virol 77: 6394-6404, 2003.

9. Palmer DH, Young LS and Mautner V: Cancer gene-therapy: clinical trials. Trends Biotechnol 24: 76-82, 2006.

10. Moolten FL: Tumor chemosensitivity conferred by inserted herpes thymidine kinase genes: paradigm for a prospective cancer control strategy. Cancer Res 46: 5276-5281, 1986.

11. Mesnil $\mathrm{M}$ and Yamasaki $\mathrm{H}$ : Bystander effect in herpes simplex virus-thymidine kinase/ganciclovir cancer gene therapy: role of gap-junctional intercellular communication. Cancer Res 60: 3989-3999, 2000.

12. Black ME, Newcomb TG, Wilson HM and Loeb LA: Creation of drug-specific herpes simplex virus type 1 thymidine kinase mutants for gene therapy. Proc Natl Acad Sci USA 93: 3525-3529, 1996.

13. Black ME, Kokoris MS and Sabo P: Herpes simplex virus-1 thymidine kinase mutants created by semi-random sequence mutagenesis improve prodrug-mediated tumor cell killing. Cancer Res 61: 3022-3026, 2001.

14. Blumenthal M, Skelton D, Pepper KA, Jahn T, Methangkool E and Kohn DB: Effective suicide gene therapy for leukemia in a model of insertional oncogenesis in mice. Mol Ther 15: 183-192, 2007.

15. Yaghoubi SS, Barrio JR, Namavari M, Satyamurthy N, Phelps ME, Herschman HR, et al: Imaging progress of herpes simplex virus type 1 thymidine kinase suicide gene therapy in living subjects with positron emission tomography. Cancer Gene Ther 12: 329-339, 2005.

16. Gambhir SS, Herschman HR, Cherry SR, et al: Imaging transgene expression with radionuclide imaging technologies. Neoplasia 2: 118-138, 2000.

17. Tjuvajev JG, Finn R, Watanabe K, et al: Noninvasive imaging of herpes virus thymidine kinase gene transfer and expression: a potential method for monitoring clinical gene therapy. Cancer Res 56: 4087-4095, 1996.

18. Tjuvajev JG, Doubrovin M, Akhurst T, et al: Comparison of radiolabeled nucleoside probes (FIAU, FHBG, and FHPG) for PET imaging of HSV1-tk gene expression. J Nucl Med 43: 1072-1083, 2002 . 
19. Alauddin MM, Shahinian A, Gordon EM, Bading JR and Conti PS: Preclinical evaluation of the penciclovir analog 9-(4[(18)F]fluoro-3-hydroxymethylbutyl)guanine for in vivo measurement of suicide gene expression with PET. J Nucl Med 42: 1682-1690, 2001.

20. Kim SJ, Doudet DJ, Studenov AR, et al: Quantitative micro positron emission tomography (PET) imaging for the in vivo determination of pancreatic islet graft survival. Nat Med 12: 1423-1428, 2006.

21. Shin O, Kim SJ, Lee WI, Kim JY and Lee H: Effective transduction by self-complementary adeno-associated viruses of human dendritic cells with no alteration of their natural characteristics. J Gene Med 10: 762-769, 2008.

22. Jun EJ, Nam YR, Ahn J, et al: Antiviral potency of a siRNA targeting a conserved region of coxsackievirus A24. Biochem Biophys Res Commun 376: 389-394, 2008.

23. Kim SJ, Lee WI, Lee YS, et al: Effective relief of neuropathic pain by adeno-associated virus-mediated expression of a small hairpin RNA against GTP cyclohydrolase 1. Mol Pain 18: 67, 2009.

24. Yaghoubi SS and Gambhir SS: PET imaging of herpes simplex virus type 1 thymidine kinase (HSV1-tk) or mutant HSV1-sr39tk reporter gene expression in mice and humans using [18F]FHBG. Nat Protoc 1: 3069-3075, 2006.

25. Bankiewicz KS, Forsayeth J, Eberling JL, et al: Long-term clinical improvement in MPTP-lesioned primates after gene therapy with AAV-hAADC. Mol Ther 14: 564-570, 2006.

26. Kota J, Chivukula RR, O'Donnell KA, et al: Therapeutic micro RNA delivery suppresses tumorigenesis in a murine liver cancer model. Cell 137: 1005-1017, 2009.

27. Garin MI, Garrett E, Tiberghien P, et al: Molecular mechanism for ganciclovir resistance in human $\mathrm{T}$ lymphocytes transduced with retroviral vectors carrying the herpes simplex virus thymidine kinase gene. Blood 97: 122-129, 2001.
28. Jang SJ, Kang JH, Lee LJ, et al: Application of bioluminescence imaging to therapeutic intervention of herpes simplex virus type I-thymidine kinase/ganciclovir in glioma. J Nucl Med 51: 1012, 2010.

29. Chin FT, Namavari M, Levi J, et al: Semiautomated radiosynthesis and biological evaluation of [18F]FEAU: a novel PET imaging agent for HSV1-tk/sr39tk reporter gene expression. Mol Imaging Biol 10: 82-91, 2008.

30. Cheng YC, Dutschman G, Fox JJ, Watanabe KA and Machida H: Differential activity of potential antiviral nucleoside analogs on herpes simplex virus-induced and human cellular thymidine kinases. Antimicrob Agents Chemother 20: 420-423, 1981.

31. Zacchigna S, Zentilin L, Morini M, et al: AAV-mediated gene transfer of tissue inhibitor of metalloproteinases-1 inhibits vascular tumor growth and angiogenesis in vivo. Cancer Gene Ther 11: 73-80, 2004

32. Noro T, Miyake K, Suzuki-Miyake N, et al: Adeno-associated viral vector-mediated expression of endostatin inhibits tumor growth and metastasis in an orthotropic pancreatic cancer model in hamsters. Cancer Res 64: 7486-7490, 2004.

33. Ponnazhagan S, Mahendra G, Kumar S, et al: Adeno-associated virus 2-mediated antiangiogenic cancer gene therapy: long-term efficacy of a vector encoding angiostatin and endostatin over vectors encoding a single factor. Cancer Res 64: 1781-1787, 2004.

34. Subramanian IV, Bui Nguyen TM, Truskinovsky AM, Tolar J, Blazar BR and Ramakrishnan S: Adeno-associated virusmediated delivery of a mutant endostatin in combination with carboplatin treatment inhibits orthotopic growth of ovarian cancer and improves long-term survival. Cancer Res 66: 4319-4328, 2006.

35. Cai KX, Tse LY, Leung C, Tam PK, Xu R and Sham MH: Suppression of lung tumor growth and metastasis in mice by adeno-associated virus-mediated expression of vasostatin. Clin Cancer Res 14: 939-949, 2008. 